\title{
Differential invariants of second-order ordinary differential equations
}

\author{
M. Eugenia Rosado \\ Departamento de Matemática Aplicada \\ ETSAM, UPM \\ Madrid, Spain
}

June 2011 


\section{Second-order ordinary differential equations}

Let $M$ be a manifold, $\operatorname{dim} M=n$.

Let $p: \mathbb{R} \times M \rightarrow \mathbb{R}$ be the natural projection. Let $p^{k}: J^{k}(\mathbb{R}, M) \rightarrow \mathbb{R}$ be the $k$-jet bundle of $p, J^{0}(\mathbb{R}, M)=\mathbb{R} \times M$, with natural projections

$$
p_{h}^{k}: J^{k}(\mathbb{R}, M) \rightarrow J^{h}(\mathbb{R}, M), \quad k \geq h .
$$

Coordinates induced by $\left(x^{i}\right)$ on $J^{2}(\mathbb{R}, M)$ :

$$
\left(t, x^{i} ; \dot{x}^{i}, \ddot{x}^{i}\right), 1 \leq i \leq n .
$$

A second-order ordinary differential equation (SODE)

$$
\ddot{x}^{i}=F^{i}\left(t, x^{i}, \dot{x}^{i}\right), \quad F^{i} \in C^{\infty}\left(J^{1}(\mathbb{R}, M)\right), 1 \leq i \leq n,
$$

can be viewed as a section $\sigma$ of the natural projection $p_{1}^{2}: J^{2}(\mathbb{R}, M) \rightarrow J^{1}(\mathbb{R}, M)$, by setting

$$
\ddot{x}^{i} \circ \sigma=F^{i} \text {. }
$$




\section{Vertical automorphisms}

Every $p$-vertical automorphism $\Phi$ of $p, \Phi(t, x)=(t, \phi(t, x))$, induces, for $r \geq 1$, a diffeomorphism

$$
\begin{aligned}
& J^{r}(\mathbb{R}, M) \stackrel{\Phi^{(r)}}{\longrightarrow} J^{r}(\mathbb{R}, M), \quad \Phi^{(r)}\left(j_{t}^{r} \gamma\right)=j_{t}^{r}\left(\Phi \circ j^{0} \gamma\right), \\
& \downarrow \quad \downarrow \quad \forall \gamma \in C^{\infty}(\mathbb{R}, M)
\end{aligned}
$$

$\mathbb{R} \times M \quad \stackrel{\Phi}{\longrightarrow} \mathbb{R} \times M$,

$\left(p_{1}^{2}\right)^{r}: J^{2}\left(p_{1}^{2}\right) \rightarrow J^{1}(\mathbb{R}, M), r$-jet bundle of $p_{1}^{2}: J^{2}(\mathbb{R}, M) \rightarrow J^{1}(\mathbb{R}, M)$.

Every $\Phi \in \operatorname{Aut}^{v}(p)$ induces a transformation $\left(\Phi^{(2)}\right)^{(r)}$ of $J^{r}\left(p_{1}^{2}\right)$ given by

$$
\left(\Phi^{(2)}\right)^{(r)}\left(j_{\xi}^{r} \sigma\right)=j_{\Phi^{(1)}(\xi)}^{r}(\Phi \cdot \sigma)
$$

where

$$
\Phi \cdot \sigma=\Phi^{(2)} \circ \sigma \circ\left(\Phi^{(1)}\right)^{-1}
$$




\section{The definition of a differential invariant}

A smooth function $\mathcal{I}: \mathcal{U} \subseteq J^{r}\left(p_{1}^{2}\right) \rightarrow \mathbb{R}$ is a differential invariant of order $r$ with respect to the group $\operatorname{Aut}^{v}(p)$ if

$$
\mathcal{I} \circ\left(\Phi^{(2)}\right)^{(r)}=\mathcal{I}, \quad \forall \Phi \in \operatorname{Aut}^{v}(p) .
$$

For a given SODE $\sigma$ on $M$, we set

$$
I(\sigma, \xi)=\mathcal{I}\left(j_{\xi}^{r} \sigma\right), \quad \xi \in J^{1}(\mathbb{R}, M) .
$$

The invariance condition reads as:

$$
I\left(\Phi \cdot \sigma, \Phi^{(1)}(\xi)\right)=I(\sigma, \xi)
$$

$\forall \xi \in J^{1}(\mathbb{R}, M)$ and $\forall \Phi \in \operatorname{Aut}^{v}(p)$ 


\section{Infinitesimal differential invariant}

If $\Phi_{t} \in \operatorname{Aut}^{v}(p)$ is the flow of a $p$-vertical $X \in \mathfrak{X}(\mathbb{R} \times M)$, then

- $\Phi_{t}^{(2)}$ flow of a $p^{2}$-vertical $X^{(2)} \in \mathfrak{X}\left(J^{2}(\mathbb{R}, M)\right)$,

- $\left(\Phi_{t}^{(2)}\right)^{(r)}$ flow of a vector field $\left(X^{(2)}\right)^{(r)} \in \mathfrak{X}\left(J^{r}\left(p_{1}^{2}\right)\right)$.

Every differential invariant $\mathcal{I}$ of order $r$ satisfies

$$
\left(X^{(2)}\right)^{(r)}(\mathcal{I})=0, \quad \text { for all } p \text {-vertical } X \in \mathfrak{X}(\mathbb{R} \times M) .
$$

Let $\mathcal{D}^{(r)}$ be the distribution on $J^{r}\left(p_{1}^{2}\right)$ spanned by all the $r$-jet prolongations $\left(X^{(2)}\right)^{(r)}$ of $p$-vertical vector fields $X \in \mathfrak{X}(\mathbb{R} \times M)$.

\section{Theorem}

$$
\operatorname{rank} \mathcal{D}^{(2)}= \begin{cases}\frac{1}{2} n\left(3 n^{2}+11 n+10\right), & n \geq 2 \\ 11, & n=1\end{cases}
$$




\section{Goal of the talk}

- GOAL: To determine second-order invariants of a SODE. The only zero- and first-order invariants are $f(t), f \in C^{\infty}(\mathbb{R})$.

- For $n \leq 2$, it is possible to determine a explicit basis for second-order differential invariants of a SODE.

D. D. Kosambi, Systems of differential equations of the second order, Quart. J. Math. Oxford 6 (1935), 1-12.

- For $n \geq 3$, the main result states that invariant functions factor through the curvature mapping attached to each SODE, which almost coincides with the torsion tensor of the Chern connection.

- We also remark the similarity between this result and the geometric version of the Utiyama theorem in gauge theories.

D. Bleecker, Gauge Theory and Variational Principles, Addison-Wesley Publishing Company, Inc., Reading, MA, 1981.

R. Utiyama, Invariant Theoretical Interpretation of Interaction, Phys. Rev. 101 (1956), 1597-1607. 


\section{The splitting induced by a SODE (1)}

As is known, $p_{0}^{1}: J^{1}(\mathbb{R}, M) \rightarrow \mathbb{R} \times M$ is an affine bundle modelled over $p^{*} T M$ where

$$
p^{\prime}: \mathbb{R} \times M \rightarrow M, \quad p^{\prime}(t, x)=x .
$$

The following exact sequence holds:

$$
0 \rightarrow\left(p^{\prime} \circ p_{0}^{1}\right)^{*} T M \stackrel{\varepsilon}{\rightarrow} V\left(p_{0}^{1}\right) \rightarrow T\left(J^{1}(\mathbb{R}, M)\right) \stackrel{\left(p_{0}^{1}\right)_{*}}{\rightarrow}\left(p_{0}^{1}\right)^{*} T(\mathbb{R} \times M) \rightarrow 0
$$

where $\varepsilon$ is defined by the directional derivative and $V\left(p_{0}^{1}\right)$ denotes the vector subbundle of $p_{0}^{1}$-vertical vectors.

We look for a section of $\left(p_{0}^{1}\right)_{*}$ in such a way

that the exact sequence splits. 


\section{The splitting induced by a SODE (2)}

\section{Dynamical flow associated to a SODE}

Every SODE $\sigma$ defines a vector field $X^{\sigma} \in \mathfrak{X}\left(J^{1}(\mathbb{R}, M)\right)$, called the dynamical flow associated to $\sigma$, as follows:

$$
\left(X^{\sigma}\right)_{\xi}=\left(j^{1} \gamma\right)_{*}\left(\frac{d}{d t}\right)_{t_{0}}, \quad \forall \xi \in\left(p^{1}\right)^{-1}\left(t_{0}\right),
$$

where by $\gamma^{i}=x^{i} \circ \gamma, 1 \leq i \leq n$, is the only solution to $\ddot{x}^{i}=F^{i}$ satisfying

$$
\gamma^{i}\left(t_{0}\right)=x^{i}(\xi), \quad \frac{d \gamma^{i}}{d t}=\dot{x}^{i}(\xi) .
$$

In coordinates, we have

$$
X^{\sigma}=\frac{\partial}{\partial t}+\dot{x}^{i} \frac{\partial}{\partial x^{i}}+F^{i} \frac{\partial}{\partial \dot{x}^{i}} .
$$




\section{The splitting induced by a SODE (3)}

The Lie derivative of the fundamental tensor field

$$
J=\omega^{i} \otimes \frac{\partial}{\partial \dot{x}^{i}}, \quad \omega^{i}=d x^{i}-\dot{x}^{i} d t,
$$

on $J^{1}(\mathbb{R}, M)$ along $X^{\sigma}$ is

$$
L_{X^{\sigma}} J=-\omega^{i} \otimes X_{i}^{\sigma}+\omega^{j} \otimes \frac{\partial}{\partial \dot{x}^{j}}, \quad \omega^{j}=d \dot{x}^{j}-F^{j} d t-\frac{1}{2} \frac{\partial F^{j}}{\partial \dot{x}^{i}} \omega^{i} .
$$

Then $T\left(J^{1}(\mathbb{R}, M)\right)=T^{0} \oplus T^{-} \oplus T^{+}$, where

$$
\begin{aligned}
& T^{0}=\operatorname{ker}\left(L_{X^{\sigma}} J\right)=\left\langle X^{\sigma}\right\rangle, \\
& T^{-}=\operatorname{ker}\left(L_{X^{\sigma}} J+I\right)=\left\langle X_{i}^{\sigma}\right\rangle, \quad X_{i}^{\sigma}=\frac{\partial}{\partial x^{\prime}}+\frac{1}{2} \frac{\partial F^{j}}{\partial \dot{x}^{\prime}} \frac{\partial}{\partial \dot{x}^{\jmath}}, \\
& T^{+}=\operatorname{ker}\left(L_{X^{\sigma}} J-I\right)=V\left(p_{0}^{1}\right)=\left\langle\frac{\partial}{\partial \dot{x}^{i}}\right\rangle .
\end{aligned}
$$




\section{The splitting induced by a SODE (4)}

The inverse mapping of the isomorphism

$$
\left.\left(p_{0}^{1}\right)_{*}\right|_{T^{0} \oplus T^{-}}: T^{0} \oplus T^{-} \stackrel{\cong}{\longrightarrow}\left(p_{0}^{1}\right)^{*} T(\mathbb{R} \times M),
$$

determines a section $H^{\sigma}$ of $\left(p_{0}^{1}\right)_{*}: T\left(J^{1}(\mathbb{R}, M)\right) \rightarrow\left(p_{0}^{1}\right)^{*} T(\mathbb{R} \times M)$ given by

$$
H^{\sigma}=d t \otimes X^{\sigma}+\omega^{i} \otimes X_{i}^{\sigma} .
$$

Every $X$ in $T\left(J^{1}(\mathbb{R}, M)\right)$ can uniquely be written as $X=X^{v}+X^{h}$,

$$
\begin{aligned}
& X^{h}=H^{\sigma}\left(\left(p_{0}^{1}\right)_{*} X\right) \in T^{0} \oplus T^{-}, \\
& X^{v}=X-X^{h} \in V\left(p_{0}^{1}\right) .
\end{aligned}
$$




\section{The curvature of the splitting}

The curvature form $K^{\sigma} \in \wedge^{2} T^{*}\left(J^{1}(\mathbb{R}, M)\right) \otimes V\left(p_{0}^{1}\right)$, of the splitting $H^{\sigma}$,

$$
K^{\sigma}(X, Y)=\left[X^{h}, Y^{h}\right]^{v}, \quad \forall X, Y \in \mathfrak{X}\left(J^{1}(\mathbb{R}, M)\right) .
$$

- From the formula for $K^{\sigma}$ in local coordinates we have:

$K^{\sigma} \in\left(p_{0}^{1}\right)^{*} \wedge^{2} T^{*}(\mathbb{R} \times M) \otimes V\left(p_{0}^{1}\right)$.

- CURVATURE MAPPING

$$
\begin{aligned}
\mathcal{K}: \mathcal{J}^{2}\left(p_{1}^{2}\right) & \rightarrow\left(p_{0}^{1}\right)^{*} \wedge^{2} T^{*}(\mathbb{R} \times M) \otimes V\left(p_{0}^{1}\right), \\
\mathcal{K}\left(j_{\xi}^{2} \sigma\right) & =\left(K^{\sigma}\right)_{\xi} .
\end{aligned}
$$

- Let $\nabla^{\sigma}$ be the Chern connection attached to $\sigma$ and let $T^{\sigma}$ be the torsion tensor field of $\nabla^{\sigma}$. We have:

$$
T^{\sigma}=K^{\sigma}+d t \wedge \omega^{i} \otimes X_{i}^{\sigma} .
$$




\section{Functoriality of the Chern connection}

The Chern connection $\nabla^{\sigma}$ is functorial with respect to $\operatorname{Aut}^{v}(p)$; i.e.,

$$
\Phi \cdot \nabla^{\sigma}=\nabla^{\Phi \cdot \sigma}, \quad \forall \Phi \in \operatorname{Aut}^{v}(p),
$$

where $\Phi \cdot \nabla^{\sigma}$ is the linear connection defined by,

$$
\left(\Phi \cdot \nabla^{\sigma}\right)_{X} Y=\Phi^{(1)} \cdot\left(\left(\nabla^{\sigma}\right)_{\left(\Phi^{(1)}\right)^{-1} \cdot X}\left(\left(\Phi^{(1)}\right)^{-1} \cdot Y\right)\right)
$$

$\forall X, Y \in \mathfrak{X}\left(J^{1}(\mathbb{R}, M)\right)$ and $\Phi \cdot \sigma$ is the SODE given by,

$$
\Phi \cdot \sigma=\Phi^{(2)} \circ \sigma \circ\left(\Phi^{(1)}\right)^{-1} .
$$




\section{Second-order invariants}

$$
\left(p_{1}^{2}\right)^{2}: J^{2}\left(p_{1}^{2}\right) \rightarrow J^{1}(\mathbb{R}, M), 2 \text {-jet bundle of } p_{1}^{2}: J^{2}(\mathbb{R}, M) \rightarrow J^{1}(\mathbb{R}, M) \text {. }
$$

\section{Theorem}

Every second-order differential invariant $\mathcal{I}: \mathcal{J}^{2}\left(p_{1}^{2}\right) \rightarrow \mathbb{R}$ with respect to $\operatorname{Aut}^{\vee}(p)$ factors uniquely through the curvature mapping: $\mathcal{I}=\overline{\mathcal{I}} \circ \mathcal{K}$,

$$
\begin{aligned}
& J^{2}\left(p_{1}^{2}\right) \stackrel{\mathcal{K}}{\longrightarrow}\left(p_{0}^{1}\right)^{*} \wedge^{2} T^{*}(\mathbb{R} \times M) \otimes V\left(p_{0}^{1}\right) \\
& \mathcal{I} \downarrow \quad \bar{L}
\end{aligned}
$$

where $\overline{\mathcal{I}}:\left(p_{0}^{1}\right)^{*} \wedge^{2} T^{*}(\mathbb{R} \times M) \otimes V\left(p_{0}^{1}\right) \rightarrow \mathbb{R}$ is an invariant smooth function under the natural action of $\operatorname{Aut}^{v}(p)$. 


\section{Concluding remarks}

As $\mathcal{D}^{(2)}$ is involutive, the number of functionally independent second-order differential invariants is

$$
\operatorname{dim} J^{2}\left(p_{1}^{2}\right)-\operatorname{rank} \mathcal{D}^{(2)}= \begin{cases}\frac{1}{2} n^{2}(n-1)+1, & n \geq 2 \\ 2, & n=1\end{cases}
$$

An endomorphism $\tilde{K}^{\sigma}:\left(p^{\prime} \circ p_{0}^{1}\right)^{*} T M \rightarrow\left(p^{\prime} \circ p_{0}^{1}\right)^{*} T M$ is defined:

$$
\tilde{K}^{\sigma}=\left.\varepsilon^{-1} \circ i_{X^{\sigma}} K^{\sigma}\right|_{T^{-}} \circ\left(\iota_{1}\right)^{-1} \circ \iota_{2}
$$

where $\iota_{1}, \iota_{2}$ are the isomorphisms:

$$
\begin{gathered}
\iota_{1}: T^{-} \stackrel{\cong}{\longrightarrow}\left(p_{0}^{1}\right)^{*} T(\mathbb{R} \times M) /\left(p_{0}^{1}\right)_{*} T^{0}, \\
\iota_{2}:\left(p^{\prime} \circ p_{0}^{1}\right)^{*} T M \hookrightarrow\left(p_{0}^{1}\right)^{*} T(\mathbb{R} \times M) \rightarrow\left(p_{0}^{1}\right)^{*} T(\mathbb{R} \times M) /\left(p_{0}^{1}\right)_{*} T^{0} .
\end{gathered}
$$

We have: $\tilde{K}^{\sigma}\left(\partial / \partial x^{j}\right)=-P_{j}^{h} \partial / \partial x^{h}$. The coefficients of the characteristic polynomial of $\tilde{K}^{\sigma}$ determine $n$ second-order invariants. 


\section{References}

G. B. Byrnes, A complete set of Bianchi identities for tensor fields along the tangent bundle projection, J. Phys. A: Math. Gen. 27 (1994), 6617-6632.

S.-S. Chern, Sur la géométrie d'un système d'équations différentielles du second ordre, Bull. Sci. Math. 63 (1939), 206-212.

M. Crampin, E. Martínez, and W. Sarlet, Linear connections for systems of second-order ordinary differential equations, Ann. Inst. H. Poincaré Phys. Théor. 65 (1996), no. 2, 223-249.

A. Kumpera, Invariants différentiels d'un pseudogroupe de Lie. I, II, J. Differential Geom. 10 (1975), 289-345, 347-416.

E. Massa, E. Pagani, Jet bundle geometry, dynamical connections, and the inverse problem of Lagrangian mechanics, Ann. Inst. H. Poincaré Phys. Théor. 61 (1994), no. 1, 17-62. 


\section{Formulas in local coordinates}

\section{The curvature form}

The curvature form:

$$
\begin{gathered}
K^{\sigma}=-\left(P_{j}^{h} d t \wedge \omega^{j}+\sum_{i<j} T_{i j}^{h} \omega^{i} \wedge \omega^{j}\right) \otimes \frac{\partial}{\partial \dot{x}^{h}} \\
T_{i j}^{h}=\frac{1}{2}\left(\frac{\partial^{2} F^{h}}{\partial x^{i} \partial \dot{x}^{j}}-\frac{\partial^{2} F^{h}}{\partial x^{j} \partial \dot{x}^{i}}\right)+\frac{1}{4}\left(\frac{\partial F^{r}}{\partial \dot{x}^{i}} \frac{\partial^{2} F^{h}}{\partial \dot{x}^{r} \partial \dot{x}^{j}}-\frac{\partial F^{r}}{\partial \dot{x}^{j}} \frac{\partial^{2} F^{h}}{\partial \dot{x}^{r} \partial \dot{x}^{i}}\right), \\
P_{j}^{h}=\frac{1}{2} X^{\sigma}\left(\frac{\partial F^{h}}{\partial \dot{x}^{j}}\right)-\frac{\partial F^{h}}{\partial x^{j}}-\frac{1}{4} \frac{\partial F^{r}}{\partial \dot{x}^{j}} \frac{\partial F^{h}}{\partial \dot{x}^{r}} .
\end{gathered}
$$




\section{Formulas in local coordinates}

\section{Chern connection}

Given a SODE $\sigma$, the Chern connection $\nabla^{\sigma}$ as defined by [2], [5], is locally given by

$$
\begin{array}{lll}
\nabla_{X^{\sigma}}^{\sigma} X^{\sigma}=0, & \nabla_{X^{\sigma}}^{\sigma} X_{i}^{\sigma}=-\frac{1}{2} \frac{\partial F^{j}}{\partial \dot{x}^{\prime}} X_{j}^{\sigma}, & \nabla_{X^{\sigma}}^{\sigma} \frac{\partial}{\partial \dot{x}^{\prime}}=-\frac{1}{2} \frac{\partial F^{j}}{\partial \dot{x}^{\prime}} \frac{\partial}{\partial \dot{x}^{j}}, \\
\nabla_{X_{i}^{\sigma}}^{\sigma} X^{\sigma}=0, & \nabla_{X_{j}^{\sigma}}^{\sigma} X_{i}^{\sigma}=-\frac{1}{2} \frac{\partial^{2} F^{k}}{\partial \dot{x}^{\prime} \partial \dot{x}^{j}} X_{k}^{\sigma}, & \nabla_{X_{i}^{\sigma}}^{\sigma} \frac{\partial}{\partial \dot{x}^{j}}=-\frac{1}{2} \frac{\partial^{2} F^{k}}{\partial \dot{x}^{i} \partial \dot{x}^{j}} \frac{\partial}{\partial \dot{x}^{k}}, \\
\nabla_{\frac{\partial}{\partial \dot{x}^{\prime}}}^{\sigma} X^{\sigma}=0, & \nabla_{\frac{\partial}{\partial \dot{x}^{\prime}}}^{\sigma} X_{j}^{\sigma}=0, & \nabla_{\frac{\partial}{\partial \dot{x}^{\prime}}}^{\sigma} \frac{\partial}{\partial \dot{x}^{j}}=0 .
\end{array}
$$




\section{Formulas in local coordinates}

\section{Zero-order invariants}

From the general formulas of jet prolongation of vector fields $X=u^{i} \frac{\partial}{\partial x^{i}}$, $u^{i} \in C^{\infty}(\mathbb{R} \times M)$, one obtains

$$
\begin{aligned}
X^{(2)} & =u^{i} \frac{\partial}{\partial x^{i}}+\left(\frac{\partial u^{i}}{\partial t}+\frac{\partial u^{i}}{\partial x^{h}} \dot{x}^{h}\right) \frac{\partial}{\partial \dot{x}^{i}} \\
& +\left(\frac{\partial^{2} u^{i}}{\partial t^{2}}+2 \frac{\partial^{2} u^{i}}{\partial t \partial x^{h}} \dot{x}^{h}+\frac{\partial^{2} u^{i}}{\partial x^{h} \partial x^{k}} \dot{x}^{h} \dot{x}^{k}+\frac{\partial u^{i}}{\partial x^{h}} \ddot{x}^{h}\right) \frac{\partial}{\partial \ddot{x}^{i}} .
\end{aligned}
$$

As the values of $u^{i}$ and its derivatives can arbitrarily be taken at a given point $j_{t}^{2} \gamma \in J^{2}(\mathbb{R}, M)$, one has

$$
\mathcal{D}^{(0)}=\left\langle\frac{\partial}{\partial x^{i}}, \frac{\partial}{\partial \dot{x}^{i}}, \dot{x}^{h} \frac{\partial}{\partial \dot{x}^{i}}+\ddot{x}^{h} \frac{\partial}{\partial \ddot{x}^{i}}, \frac{\partial}{\partial \ddot{x}^{i}}, 2 \dot{x}^{h} \frac{\partial}{\partial \ddot{x}^{i}}, \dot{x}^{h} \dot{x}^{k} \frac{\partial}{\partial \ddot{x}^{i}}\right\rangle=\left\langle\frac{\partial}{\partial x^{i}}, \frac{\partial}{\partial \dot{x}^{i}}, \frac{\partial}{\partial \ddot{x}^{i}}\right\rangle .
$$

The only differential invariants of order 0 are the functions in $\left(p^{2}\right)^{*} C^{\infty}(\mathbb{R})$. 


\section{Formulas in local coordinates}

\section{First-order invariants}

By collecting the derivatives of the functions $u^{i}$ in $\left(X^{(2)}\right)^{(1)}$ we have

$$
\begin{aligned}
\left(X^{(2)}\right)^{(1)} & =u^{r} \frac{\partial}{\partial x^{r}}+\frac{\partial u^{r}}{\partial t} \chi_{t}^{r}+\frac{\partial u^{r}}{\partial x^{a}} \chi_{a}^{r} \\
& +\frac{\partial^{2} u^{r}}{\partial t^{2}} \chi_{t t}^{r}+\frac{\partial^{2} u^{r}}{\partial t \partial x^{a}} \chi_{t a}^{r}+\sum_{a \leq b} \frac{\partial^{2} u^{r}}{\partial x^{a} \partial x^{b}} \chi_{a b}^{r} \\
& +\frac{\partial^{3} u^{r}}{\partial t^{3}} \chi_{t t t}^{r}+\frac{\partial^{3} u^{r}}{\partial t^{2} \partial x^{a}} \chi_{t t a}^{r}+\sum_{a \leq b} \frac{\partial^{3} u^{r}}{\partial t \partial x^{a} \partial x^{b}} \chi_{t, a b}^{r}+\sum_{a \leq b \leq c} \frac{\partial^{3} u^{r}}{\partial x^{a} \partial x^{b} \partial x^{c}} \chi_{a b c}^{r} .
\end{aligned}
$$

for certain $\chi_{t}^{r}, \chi_{a}^{r}, \chi_{t t}^{r}, \chi_{t a}^{r}, \chi_{a b}^{r}, \chi_{t t t}^{r}, \chi_{t t a}^{r}, \chi_{t a b}^{r}, \chi_{a b c}^{r} \in \mathfrak{X}\left(J^{1}\left(p_{1}^{2}\right)\right)$. Is is proved that

$$
\begin{aligned}
\mathcal{D}^{(1)} & =\left\langle\partial / \partial x^{r}, \chi_{t}^{r}, \chi_{a}^{r}, \chi_{t t}^{r}, \chi_{t a}^{r}, \chi_{a b}^{r}, \chi_{t t t}^{r}, \chi_{t t a}^{r}, \chi_{t a b}^{r}, \chi_{a b c}^{r}\right\rangle \\
& =\left\langle\partial / \partial x^{r}, \partial / \partial \dot{x}^{r}, \partial / \partial \ddot{x}^{r}, \partial / \partial \ddot{x}_{t}^{r}, \partial / \partial \ddot{x}_{a}^{r}, \partial / \partial \ddot{x}_{\dot{a}}^{r}\right\rangle .
\end{aligned}
$$

The first-order differential invariants are $f(t), f \in C^{\infty}(\mathbb{R})$. 


\section{Formulas in local coordinates}

\section{The curvature mapping}

Equations of the curvature mapping

$$
\begin{aligned}
t \circ \mathcal{K} & =t, x^{i} \circ \mathcal{K}=x^{i}, \dot{x}^{i} \circ \mathcal{K}=\dot{x}^{i}, \\
y_{a}^{i} \circ \mathcal{K} & =-\frac{1}{2}\left(\ddot{x}_{t \dot{a}}^{i}+\dot{x}^{h} \ddot{x}_{h \dot{a}}^{i}+\ddot{x}^{h} \ddot{x}_{\dot{h} \dot{a}}^{i}\right)+\ddot{x}_{a}^{i}+\frac{1}{4} \ddot{x}_{\dot{a}}^{k} \ddot{x}_{\dot{k}}^{i}, \\
y_{a b}^{k} \circ \mathcal{K} & =-\frac{1}{2}\left(\ddot{x}_{a \dot{b}}^{k}-\ddot{x}_{b \dot{a}}^{k}+\frac{1}{2}\left(\ddot{x}_{\dot{a}}^{h} \ddot{x}_{\dot{h} \dot{b}}^{k}-\ddot{x}_{\dot{b}}^{h} \ddot{x}_{\dot{h}}^{k}\right)\right), a<b,
\end{aligned}
$$

$\left(t, x^{i}, \dot{x}^{i}, \ddot{x}^{i}, \ddot{x}_{t}^{i}, \ddot{x}_{a}^{i}, \ddot{x}_{\dot{a}}^{i}, \ddot{x}_{t t}^{i}, \ddot{x}_{t a}^{i}, \ddot{x}_{t \dot{a}}^{i}, \ddot{x}_{a \leq b}^{i}, \ddot{x}_{a b}^{i}, \ddot{x}_{\dot{a} \leq b}^{i}\right)$ induced coordinate system on $J^{2}\left(p_{1}^{2}\right)$ and

$$
\eta=\left(y_{i}^{j}(\eta)\left(d t \wedge \omega^{i}\right)_{\left(t_{0}, x_{0}\right)}+\sum_{h<i} y_{h i}^{j}(\eta)\left(\omega^{h} \wedge \omega^{i}\right)_{\left(t_{0}, x_{0}\right)}\right) \otimes\left(\frac{\partial}{\partial \dot{x}^{j}}\right)_{\xi},
$$

coordinates are introduced in $\left(p_{0}^{1}\right)^{*} \wedge^{2} T^{*}(\mathbb{R} \times M) \otimes V\left(p_{0}^{1}\right)$. 


\section{The curvature mapping}

The curvature mapping is $\operatorname{Aut}^{v}(p)$-equivariant with respect to the natural actions, i.e.,

$$
\Phi \cdot \mathcal{K}\left(j_{\xi}^{2} \sigma\right)=\mathcal{K}\left(\Phi \cdot j_{\xi}^{2} \sigma\right), \quad \forall \Phi \in \operatorname{Aut}^{v}(p)
$$

where the action on the left-hand side is defined by

$$
\Phi \cdot \eta=\left(\wedge^{2}\left(\left(\Phi^{(1)}\right)^{-1}\right)^{*} \otimes\left(\Phi^{(1)}\right)_{*}\right)(\eta),
$$

$\forall \eta \in\left(p_{0}^{1}\right)^{*} \wedge^{2} T^{*}(\mathbb{R} \times M) \otimes V\left(p_{0}^{1}\right)$, and the action on the right-hand side is defined as follows:

$$
\Phi \cdot j_{\xi}^{2} \sigma=j_{\Phi^{(1)}(\xi)}^{2}(\Phi \cdot \sigma) .
$$

